\title{
Artesanos de la salud: un concepto para pensar el hacer de campesinos sanadores en el Norte de Antioquia, Colombia
}

\section{Mauricio Múnera Gómez}

(iD) https://orcid.org/0000-0003-2300-9617

Facultad de Educación de la Universidad de Antioquia

Grupo de Investigación Somos Palabra: formación y contextos

mauricio.munera1@udea.edu.co

\section{RESUMEN}

En este artículo se desarrolla una conceptualización de la categoría artesanos de la salud para hacer referencia a hombres y mujeres que, en algunos municipios del Norte de Antioquia, Colombia, articulan manos, cabezas y corazones con el propósito de brindar un servicio a otros seres a través del desarrollo de diferentes prácticas para el cuidado de la vida. Esta escritura es una derivación de un ejercicio de investigación cualitativa fundamentada en el uso de metodologías biográficas y narrativas y en la construcción de historias de vida de campesinos sanadores. El artículo permite profundizar en las siguientes características de los artesanos de la salud: son sujetos de la experiencia, ofrecen sus servicios a las comunidades y estas validan su saber-hacer, desempeñan sus oficios en el hogar-taller como espacio de creación y desarrollan diferentes prácticas para el cuidado de la vida pensando en el servicio y no en la transmisión o la transferencia.

Palabras clave: artesanos de la salud, sanadores, prácticas para el cuidado de la vida, historias de vida, Norte de Antioquia. 


\section{Health craftsmen: A concept for thinking about the work of rural healers in the North of Antioquia, Colombia}

\section{$A B S T R A C T$}

This article develops the category of health craftsmen as a concept that refers to the men and women who, in towns of the north of Antioquia, Colombia, utilize hands, heads and hearts in order to offer a service to others, through the development of different practices for the care of life. This reflection is a result of the exercise of qualitative research based on the use of biographical and narrative methodologies for the construction of the life stories of rural healers. This article explores the following characteristics of health craftsmen: they are subjects of experience; they offer their services to the communities and the communities validate their skills; they perform their jobs in the home-workshop as a creation space; and they develop different practices for the care of life thinking about service and not about their transmission or transfer.

Keywords: health craftsmen; healers; practices for the care of life; life stories; northern Antioquia. 


\section{PRESENTACIÓN}

Este artículo es una derivación de la investigación doctoral titulada Artesanos de la salud y prácticas para el cuidado de la vida: aproximaciones a la medicina tradicional en el Norte de Antioquia, desarrollada en la línea de investigación Ciencias, Saberes y Técnicas del doctorado en Ciencias Humanas y Sociales de la Universidad Nacional de Colombia, sede Medellín. Lo que se presenta en estas páginas son algunas comprensiones alrededor de la categoría artesanos de la salud, propuesta en la investigación como una posibilidad para hacer alusión a algunos sujetos que se han dedicado a los procesos de atención de la salud y la enfermedad en comunidades campesinas del Norte de Antioquia, Colombia.

El texto se encuentra dividido en tres partes. La primera es de contextualización, en esta se expone la pregunta, los participantes, el contexto y la metodología de investigación. La segunda es de conceptualización, aquí se lleva a cabo la construcción de los conceptos sanador y artesanos de la salud a la luz del trabajo de campo desarrollado. La tercera es de conclusión, en esta se presentan algunas ideas de análisis y de cierre frente al tema del artículo.

\section{CONTEXTUALIZACIÓN}

La investigación doctoral a la que se hace alusión en este artículo estuvo orientada por la siguiente pregunta de investigación: ¿cuáles son los acontecimientos, las experiencias y las situaciones que hacen que un campesino devenga sanador y artesano de la salud? ${ }^{1}$. Para avanzar en esta comprensión, el trabajo de pesquisa se fundamentó en la apropiación y la puesta en marcha de metodologías biográficas y narrativas tales como relatos de experiencias, círculos de palabra y participación

Cabe aclarar que en este artículo no se va a profundizar en aquellas trayectorias que explican cómo un campesino deviene sanador. 
en terapéuticas, a partir de las cuales fue posible profundizar en las historias de vida de ocho sanadores que, en los municipios de San Pedro de los Milagros, Belmira, Entrerríos, Don Matías, Santa Rosa de Osos y San José de la Montaña, del departamento de Antioquia, han articulado la realización de tareas agrarias y pecuarias con el ejercicio de diferentes terapéuticas orientadas al cuidado y la recuperación de la salud.

En el siguiente organizador gráfico se presentan los nombres de los participantes de la investigación, los municipios en los que viven y las prácticas para el cuidado de la vida que desarrollan²:

\section{Figura 1. Participantes de la investigación y prácticas para el cuidado de la vida ${ }^{3}$}

\begin{tabular}{|l|l|}
\hline $\begin{array}{l}\text { Nombre, edad y } \\
\text { ubicación del sanador }\end{array}$ & Prácticas para el cuidado de la vida \\
\hline $\begin{array}{l}\text { Berta Inés Avendaño } \\
\text { Agudelo }\end{array} 90$ años & Partería \\
Santa Rosa de Osos & $\begin{array}{l}\text { Esta práctica reivindica el significado y la importancia } \\
\text { de las mujeres como guardianas de la existencia humana, } \\
\text { gracias a su desempeño como parteras o comadronas. } \\
\text { El oficio de las mujeres implica el acompañamiento de } \\
\text { las mujeres durante el embarazo, el parto y el puerperio. } \\
\text { En esta se tejen vínculos con diferentes divinidades y } \\
\text { entidades, que son invocadas para pedir protección en los } \\
\text { procesos de gestación y nacimiento de los seres humanos. }\end{array}$ \\
\hline
\end{tabular}

2 En lo que respecta a las prácticas que se caracterizaron e interpretaron en la investigación, a pesar de que estas se incluyan dentro de la nominación de artesanías de la salud, no se corresponden con un todo homogéneo; como tal: presentan particularidades simbólicas y terapéuticas propias de las trayectorias de los sujetos que las operacionalizan. El criterio fundamental para su inclusión tuvo que ver con el reconocimiento y la validación de las comunidades frente a quienes las desarrollaban. Dado que la pregunta de investigación estuvo enfocada hacia la subjetividad de los campesinos y a su devenir sanador, la inclusión de estas formas de hacer, asociadas al cuidado de la vida, se fundamentó en la interacción con los agentes comunitarios de salud y, posteriormente, en la profundización de sus modos de agenciarlas. De otro lado, estas prácticas se presentan como formas alternativas y complementarias de los procedimientos de la medicina alopática.

3 Una primera versión de este organizador gráfico fue presentada en la revista Pensamiento Actual (Múnera, 2017).

4 En coherencia con las metodologías asumidas en la investigación, y de acuerdo con el consentimiento informado, el investigador decidió usar los nombres propios, no seudónimos. 


\begin{tabular}{|c|c|}
\hline $\begin{array}{l}\text { José Leonardo Céspedes } \\
\text { Rodríguez } \\
77 \text { años } \\
\text { San Pedro de los Milagros }\end{array}$ & $\begin{array}{l}\text { Herbolaria } \\
\text { Este oficio tiene que ver con el cultivo y el uso de hierbas, } \\
\text { plantas y productos naturales, de carácter medicinal, } \\
\text { destinados al restablecimiento de funciones del cuerpo, la } \\
\text { atención de dolencias y padecimientos, la armonización } \\
\text { de espacios y situaciones y, en general, la recuperación } \\
\text { de la salud. }\end{array}$ \\
\hline $\begin{array}{l}\text { Martha Genoveva Osorno } \\
\text { Restrepo } \\
75 \text { años } \\
\text { Don Matías }\end{array}$ & $\begin{array}{l}\text { Heliocromoterapia } \\
\text { Esta práctica se fundamenta en el reconocimiento de } \\
\text { tres instancias transmisoras de energía: el sol, el color } \\
\text { y el agua, entre las cuales se da una interacción y cuya } \\
\text { sinergia deviene en un proceso terapéutico. Requiere el } \\
\text { uso de cinco botellas de vidrio de diversos colores: azul, } \\
\text { verde, amarillo, rojo y blanco. El agua se pone al sol } \\
\text { durante tres o cuatro horas y luego se consume (se puede } \\
\text { tomar en pequeñas cantidades o aplicar en el cuerpo, } \\
\text { según el color de la botella y el padecimiento que se esté } \\
\text { tratando). }\end{array}$ \\
\hline $\begin{array}{l}\text { Argemira Echeverry } \\
\text { Molina } \\
73 \text { años } \\
\text { San Pedro de los Milagros }\end{array}$ & $\begin{array}{l}\text { Oración y canalización de José Gregorio Hernández } \\
\text { Cisneros } \\
\text { Este oficio tiene que ver con la invocación y veneración } \\
\text { de José Gregorio Hernández Cisneros, médico alópata, } \\
\text { considerado el «padre de la medicina moderna en } \\
\text { Venezuela». Elnombredeestemédicoysureconocimiento } \\
\text { se extienden por diferentes territorios latinoamericanos } \\
\text { gracias a la importancia de su obra y su pensamiento } \\
\text { para la medicina científica de su país, al recuerdo de } \\
\text { su condición de hombre solidario y benefactor de los } \\
\text { más pobres, y luego de su muerte, a los innumerables } \\
\text { milagros que ha realizado en favor de muchas personas } \\
\text { que presentaron significativos padecimientos de salud. }\end{array}$ \\
\hline
\end{tabular}




\begin{tabular}{|c|c|}
\hline $\begin{array}{l}\text { Blasina del Socorro Mery } \\
\text { de Correa } \\
63 \text { años } \\
\text { San José de la Montaña }\end{array}$ & $\begin{array}{l}\text { Sobanderismo } \\
\text { Es una especialidad de la medicina tradicional que } \\
\text { enfatiza en el tratamiento de molestias y afectaciones en la } \\
\text { estructura osteomuscular de los seres humanos, es decir, } \\
\text { en lo que tiene que ver con músculos, huesos, tendones, } \\
\text { ligamentos, articulaciones y cartílagos, a través del rezo } \\
\text { o del masaje y la presión. Esta práctica también se ofrece } \\
\text { para la atención de algunos animales, especialmente, de } \\
\text { aquellos domésticos o de uso constante en las actividades } \\
\text { productivas del campo: vacas, caballos, cerdos, entre } \\
\text { otros. }\end{array}$ \\
\hline $\begin{array}{l}\text { José David } \\
\text { Múnera Ochoa } \\
53 \text { años } \\
\text { San Pedro de los Milagros }\end{array}$ & $\begin{array}{l}\text { Limpieza a través de la luz } \\
\text { Esta práctica se fundamenta en la oración como una } \\
\text { manera de establecer conexión con la fuerza superior } \\
\text { de la cual proviene la curación, e implica el uso de una } \\
\text { fotografía reciente del cuerpo del consultante y una } \\
\text { vela. El sanador toma la fotografía y la pone delante } \\
\text { de la luz de una vela. Gracias al reflejo de la luz } \\
\text { aparecen diferentes sombras en la fotografía, las cuales } \\
\text { son interpretadas por el terapeuta para construir un } \\
\text { diagnóstico. Posteriormente, se deja caer parafina sobre } \\
\text { la imagen de la fotografía, para que se ilumine con la luz } \\
\text { y así se lleve a cabo la limpieza de los padecimientos } \\
\text { físicos, mentales o emocionales del consultante. }\end{array}$ \\
\hline $\begin{array}{l}\text { Ramiro Antonio Velásquez } \\
50 \text { años } \\
\text { San José de la Montaña }\end{array}$ & $\begin{array}{l}\text { Imposición de manos } \\
\text { Este oficio guarda relación con la obra «salvadora» de } \\
\text { Jesús de Nazareth, así como con el empoderamiento } \\
\text { de determinados hombres, quienes, por su investidura, } \\
\text { tuvieron una capacidad especial para infundir fuerza, } \\
\text { energía y bendición a las personas que se acercaban } \\
\text { a ellos gracias al uso de las manos. Se reconocen dos } \\
\text { líneas: la imposición de manos como una derivación de } \\
\text { la tradición cristiana inspirada en la figura de Jesús, y el } \\
\text { tacto curativo y el reiki para la transmisión de la fuerza } \\
\text { o la energía vital. }\end{array}$ \\
\hline
\end{tabular}




\begin{tabular}{|l|l|}
\hline Jesús María Tobón Mazo & $\begin{array}{l}\text { Animería } \\
\text { Eelmira y Entrerríos }\end{array}$ \\
& $\begin{array}{l}\text { Eue tiene que ver con las dimensiones espirituales y } \\
\text { emocionales de los seres humanos y con el descanso } \\
\text { eterno de las almas de las personas, que desencarnaron } \\
\text { a causa de una enfermedad, un accidente o un acto } \\
\text { violento. El animero, durante el mes de noviembre, en la } \\
\text { medianoche, va al cementerio e invita a las almas de los } \\
\text { difuntos para que salgan con él a caminar por las calles } \\
\text { del pueblo. Mientras que el animero camina con las } \\
\text { ánimas, desde las casas las personas rezan padrenuestros } \\
\text { por el descanso eterno de sus seres queridos. }\end{array}$ \\
\hline
\end{tabular}

En lo que respecta a las metodologías biográficas y narrativas, en la investigación se abordaron las experiencias, los recorridos y las comprensiones generadas por los sujetos frente a su propia vida, a través del uso de la narración. Así, en estas metodologías se entretejen diversas formas del relato, tantísimas manifestaciones de la palabra y, en sí, de los géneros discursivos (Bajtín, 1998). El relato trae consigo la evocación de la experiencia, por ello, es otra posibilidad para vivir de nuevo la vida. Se trata de reconocer, describir e interpretar las múltiples formas de la subjetividad y, en otros términos, las distintas trayectorias de los sujetos.

Se elaboraron, entonces, biografías interpretadas (Mallimaci y Giménez Béliveau, 2006, p. 177), gracias a las cuales fueron reconstruidas voces y testimonios, las cuales derivaron en determinados conceptos y líneas de análisis. Por ello, el uso de las historias de vida no se limitó a la recopilación de datos: más bien, las acciones se encaminaron hacia un ejercicio comprensivo desde el tejido de memorias y experiencias. De esta manera, gracias a determinados episodios de esa gran historia, que es la vida misma, fue posible construir argumentos y explicaciones acerca de la condición de sanadores y artesanos de la salud, conceptos que se desarrollan en este artículo.

Para cerrar esta primera parte del texto, en el siguiente mapa se presenta la región en la cual fue realizada la investigación: 
Figura 2. Mapa de la región del Norte de Antioquia

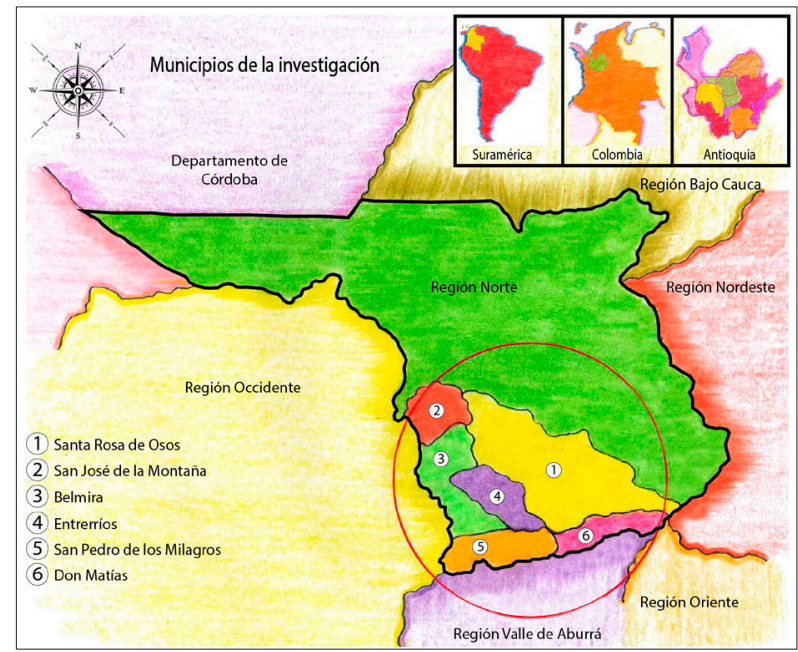

A propósito del Norte de Antioquia, es preciso mencionar que esta es una de las nueve regiones en las que se divide Antioquia como departamento. Esta región se encuentra ubicada en la Cordillera Central de Colombia y está diferenciada en dos macrozonas: Altiplano Norte y vertientes de los ríos Cauca y Nechí. Los municipios en los que se desarrolló la investigación forman parte de la primera macrozona y se integran en los territorios de las vertientes de los ríos Chico y Grande.

En cuanto a algunas de sus características y condiciones territoriales, el Norte de Antioquia - y, en concreto, la zona de las vertientes de los ríos Chico y Grande - se destacan por el desarrollo de actividades pecuarias y agrícolas. En lo que respecta a lo pecuario, en la zona se da el cuidado y la manutención de ganado bovino (representado en hatos lecheros de raza Holstein, en mayor medida) y porcino (aunque esta actividad económica no es tan fuerte como la anterior). Frente a lo agrícola, algunos campesinos de la zona se dedican al cultivo de productos tales como el maíz, la papa, el tomate de árbol y, en algunas zonas un poco más templadas, el plátano, la caña de azúcar y el café, entre otros.

Otro aspecto importante de la región y de la macrozona tiene que ver con la existencia del Páramo Santa Inés, lugar sagrado para la generación del agua de la que se alimentan los diferentes seres vivos del territorio y de otras regiones del departamento. De tal suerte, la región se caracteriza por la riqueza hídrica, tanto así que allí se han construido importantes proyectos hidroeléctricos, como Río Grande I y II, Guadalupe, Porce II y III. En otras palabras, es un espacio que 
se encuentra trazado por la presencia de bosques, los movimientos de ganados bovinos y la extensión de hilos de agua, que hacen de sus tierras espacios fértiles para la emergencia y el cuidado de la vida.

Finalmente, el grupo poblacional de mayor predominancia en la macrozona en la que se realizó la investigación es el mestizo. Durante el tiempo de la conquista y la colonización de nuestro continente, en esta región se destacó la presencia de grupos indígenas como los nutabes y los tahamies, así como la existencia de osos de anteojos. Sin embargo,

[...] tanto los indígenas, como los majestuosos animales desaparecieron de la zona, dejando prácticamente aniquilada la remembranza de su historia. De los osos, hoy por hoy, solo se puede encontrar una escultura a la entrada del municipio de Santa Rosa de Osos. En cuanto a los indígenas, si bien no hay muchas claridades acerca de su desaparición, algunos documentos se atreven a plantear la idea, según la cual, varios de ellos se movieron hacia las márgenes del Mar Caribe, otros tantos murieron en el proceso de conquista, algunos más fueron usados como esclavos para la explotación de las minas de oro, mezclando así su sangre con la de los colonizadores, y una parte restante fue incluida en el resguardo indígena que tuvo lugar en las tierras de Ituango (Múnera, 2019, pp. 72-73).

\section{EL CAMPESINO COMO SANADOR, EL CAMPESINO COMO ARTESANO DE LA SALUD}

Los campesinos son las personas más ricas y sabias, solo que no lo saben. Ellos son los que ven los atardeceres, los amaneceres, la lluvia; ellos ven las plantas crecer y son quienes están más cerca de Dios.

(José David Múnera Ochoa)

El trabajo de campo de la investigación, que estuvo orientado por el desarrollo de ejercicios biográficos y narrativos, entregó los conceptos de sanador y artesano de la salud, para hacer alusión a aquellos agentes de la salud comunitaria que desarrollan prácticas para el cuidado de la vida tales como la partería, la herbolaria, el sobanderismo, la oración y la canalización del espíritu de José Gregorio Hernández Cisneros, la limpieza a través de la luz, la animería, la heliocromoterapia y la imposición de manos. Sobre estos dos conceptos - en particular sobre el segundo- , se van a presentar algunos descriptores que permitirán ahondar en sus significados y en el porqué de su proposición. 
El uso de la voz sanador fue recurrente en las descripciones y los testimonios aportados por los campesinos para hacer referencia a las tareas que ellos desarrollan para cuidar la vida y atender la salud. Así, al acudir a los códigos emic (González Echevarría, 2009; Harris, 1982), es decir, a las propias formas de habla de los campesinos, se reconoció una especial preferencia por esta palabra y, según los análisis, se pueden plantear dos razones que justifican su uso.

En primer lugar, la figura de Jesús de Nazareth y las referencias que la Biblia hace de este personaje como sanador tienen una gran influencia en la autodenominación de los campesinos como sanadores. Así, las formas para explicar la enfermedad — en términos de pecado y desobediencia—y la sanación —asumida como limpieza y cambio en la vida-, las cuales se originaron en las experiencias vividas por este hombre, inciden en la configuración de las propias representaciones sobre la salud y la enfermedad de las ocho personas participantes de la investigación, así como en su preferencia por este término para nombrar y calificar sus oficios.

En segundo lugar, los campesinos muestran cierta predilección por las palabras sanador y sanación, pues reconocen que ambas hacen referencia a una atención que implica los siguientes campos de los seres humanos: biológico, mental, emocional y espiritual. Esto se relaciona con una comprensión de la sanación en los siguientes términos:

[...] la sanación es un fenómeno holístico o tridimensional que envuelve la recuperación del equilibrio, la armonía en el cuerpo, la mente y el espíritu, o entre la persona y el ambiente. No se enfoca en síntomas o enfermedades, sino que trata al ser humano en su totalidad. Desde esta perspectiva, la curación es dirigida a la persona y no a la enfermedad. Los sanadores tradicionales reciben enseñanzas especiales de sus ancestros y transmiten tradiciones curativas de una generación a la siguiente. Son reconocidos en la comunidad donde viven por su capacidad de sanación (López, Cataño, López y Velásquez, 2011, p. 289).

Como una derivación de este asunto, los campesinos no sienten mucha afinidad con la voz curandero, pues, según sus propias explicaciones, esta tiene otras connotaciones del orden de la superstición, la brujería y la hechicería. Esto último lo fundamentan en algunas de las ideas de la Iglesia católica, la cual rechaza, de manera frontal, cualquier vínculo con la magia, la adivinación, los rezos, entre otras expresiones de las culturas populares. En cuanto a la palabra curación, ellos señalan que no la usan mucho, pues tiene un carácter más inmediatista y de resultados de corta duración, mientras que la sanación está relacionada con 
un proceso más holístico y de mayor trascendencia por las dimensiones y los aspectos que integra.

Se destaca esta diferencia entre los modos de usar y significar las palabras sanador y curandero y sanación y curación por quienes compartieron sus testimonios durante el trabajo de campo, solo para aclarar las propias vías de significado a las que estos acuden. Sin embargo, hay que reconocer que estos cuatro conceptos forman parte de un mismo campo semántico, aunque la preferencia por unos u otros dependerá de las tradiciones y las trayectorias de las comunidades o de los académicos que los usen. De cualquier forma, al hablar de sanadores y curanderos se hace referencia a unos sujetos que emplean diferentes recursos terapéuticos — que no se limitan a los ofrecidos por la medicina alopática—, con el fin de sugerir y poner en marcha procedimientos curanderiles tendientes a mejorar las situaciones de desbalance y padecimiento que viven los seres humanos y otros seres animales y vegetales.

En diferentes investigaciones y análisis de casos se puede ver que algunos autores privilegian más un concepto que otro, o usan ambos para hacer referencia a sus hallazgos, descripciones y análisis. Por ejemplo, Press (1971), Campos Navarro (1997, 2016), Sharon (1980) y Ceballos Gómez $(1994,2002)$ usan la palabra curandero para hacer referencia a los oficios de determinados sujetos que, en contextos rurales o urbanos, ofrecen a las personas de sus comunidades o de otros conjuntos humanos gracias al uso de diferentes materiales y dispositivos simbólicos. Por su parte, Spector (2003) usa el término sanadores tradicionales para referirse a las formas de medicina tradicional de España. Finalmente, Sowell (2002), para exponer el caso de Miguel Perdomo Neira, usa de forma indistinta las palabras curandero y sanador en el momento de mostrar las particularidades de este agente de la salud de la Colombia decimonónica. Además, este último autor también usa las expresiones: empíricos y sanadores legos para hacer referencia al curandero mencionado.

En el caso de la investigación, una de las particularidades de los sanadores tiene que ver con que estos son capaces de dialogar con los usuarios de sus terapéuticas - escucharlos y compartirles sus miradas y consideraciones-, pues tienen todo el conocimiento cultural sobre las representaciones, los saberes y los discursos de la etiología popular de las enfermedades y de sus formas de clasificación, por lo tanto, al formar parte de las comunidades, tienen la capacidad de interpretar los modos a través de los cuales los sujetos explican, enfrentan y asumen los procesos de padecimiento. De otro lado, por su condición de «anfibios culturales», si se retoma el concepto sugerido por Mockus Sivickas, ellos pueden interpretar dife- 
rentes asuntos derivados de los sistemas médicos científicos, dialogar con ellos y sugerir redes de articulación entre unas formas «empíricas» y otras, supuestamente «científicas» para atender la salud y la enfermedad y cuidar la vida.

Esto último se puede ver en casos como el de Berta Inés Avendaño Agudelo, quien enriqueció sus saberes con los aportes dados por médicos alópatas y enfermeras en un curso de preparación de parteras, y también en el de José Leonardo Céspedes Rodríguez, pues él recibió algunas instrucciones de un ortopedista para mejorar su desempeño como sobandero. Ellos entablaron puentes de comunicación e interpretación entre los saberes heredados de sus familias y comunidades, y aquellas formas de razonamiento y acción aportadas por la biomedicina.

A continuación, se incluye la definición de anfibio cultural:

$[\ldots]$ es quien se desenvuelve solventemente en diversos contextos y al mismo tiempo posibilita una comunicación fértil entre ellos, es decir transporta fragmentos de verdad (o de moralidad) de un contexto a otro. Esa solvencia del anfibio cultural — capaz de obedecer a sistemas de reglas distintos en tiempos y en contextos diversos - hace que él deba ser un «camaleón». Por otro lado, el anfibio debe tener la capacidad de generar comunicación entre los diversos medios y tradiciones con los cuales entra en contacto. El anfibio cultural tiene que entender y traducir y comunicar, aunque sea fragmentariamente, saberes y pautas morales. Es decir, debe ser un intérprete (Mockus Sivickas, 1994, p. 127).

Entonces, el sanador campesino es un intérprete, un traductor y un comunicador entre universos discursivos y procedimentales, que han estado escindidos por diferentes razones y reglas de funcionamiento. Lamentablemente, desde los regímenes de cientificidad, este sujeto ha sido proscrito a unos escenarios marginales en los cuales se le cataloga como hechicero, primitivo, mentiroso o brujo (González Rodríguez, 2005), y este hecho ha despertado unas miradas de resistencia e invisibilización de sus oficios por unos sectores hegemónicos. Así las cosas, el sanador - el curandero o el curador popular, como también lo nombra Armus (2002) - ha creado unos propios dominios y un «modelo médico alternativo» (Campos-Navarro, 1997), en el que se articulan diferentes paradigmas y sistemas de conocimiento de gran riqueza y complejidad.

A propósito de estos paradigmas, Módena (1990) expone tres sistemas para la atención de la salud, a saber: el de las madres, que es el doméstico; el de los médicos académicos, que tiene todo un respaldo institucional desde el ámbito de la biomedicina, y el de los curanderos como tales, que está asociado a todas las medicinas tradicionales que tienen lugar en los universos populares. Desde la 
interpretación de los testimonios recogidos, se puede afirmar que los dos primeros sistemas, en algunos casos, se entremezclan en los oficios de los sanadores o los curanderos. Entonces, no se puede hablar de la existencia de un solo sistema o de la continuidad de unas formas de funcionamiento que se amparan bajo el regazo de un paradigma excluyente, al contrario, es importante considerar que los oficios de estos sujetos tienen sus raíces en formas de medicina doméstica y que, a partir de estas, se da un enriquecimiento con conceptos y técnicas de las diferentes culturas humanas, lo cual demuestra que el gran «monopolio del derecho a curar», al que ya se refirió Armus (2002), no ha de limitarse a los oficios de los profesionales de la medicina académica o facultativa.

Ahora bien, con el propósito de profundizar un poco más en los conceptos que se están abordando, es útil incluir dos definiciones provenientes de ámbitos disciplinares diferentes. La primera se origina en el contexto de la enfermería y es expuesta por López y otras investigadoras, a partir de las ideas entregadas por Spector. Para estas autoras, los sanadores tradicionales son:

[...] un fenómeno sociocultural tan antiguo como la historia del hombre. Coexisten con el desarrollo cultural y tecnológico de los pueblos [...]. Gozan del reconocimiento, la confianza y el respeto de sus respectivas comunidades. Son reconocidos por la atención personalizada, adaptada a las necesidades y expectativas de sus pacientes. De esta manera, son potentes agentes de comunicación para cuestiones sociales y de salud. Poseen mayor credibilidad que los agentes que provienen de las instituciones. Cobran fuerza en los entornos de recursos limitados porque las alternativas de curación proporcionadas son de fácil y rápido acceso (López y otros, 2011, p. 289).

La segunda definición nace en el seno de la antropología y es compartida por Zolla. En este caso, este investigador aporta el término terapeutas tradicionales, en el cual se integran los sanadores, los curanderos o los curadores. En sus palabras, estos terapeutas son vistos como:

[...] aquellas personas generalmente adultas y pertenecientes a la comunidad en donde ejercen, a la que el grupo social reconoce como dotadas de los conocimientos, habilidades o facultades para curar, que diagnostican las enfermedades conforme a una idea de la causalidad que es compartida por el grupo, y cuyo sistema de creencias, conceptos y prácticas las distinguen claramente de los terapeutas de la medicina institucional (Zolla, 1986, p. 17).

Al hacer una lectura de ambas definiciones y al establecer relación con los relatos y las explicaciones derivadas del trabajo de campo de la investigación, se 
puede notar que los sanadores o los terapeutas tradicionales gozan de un reconocimiento social brindado por las comunidades de las cuales hacen parte. Además, muchas veces sus oficios llegan hasta los lugares a los cuales la biomedicina o el sistema médico hegemónico no ha llegado por razones vinculadas con la geografía de los territorios o con las posibilidades económicas de las personas para acceder a los servicios de la medicina alopática, y este asunto los convierte en las primeras formas de atención de los padecimientos. En cuanto a las terapéuticas que llevan a cabo, estos sujetos usan diferentes materiales y recursos de origen mineral, vegetal o animal, que obtienen, por lo general, de sus entornos. También, en estos procedimientos de sanación, el arte de curar y la espiritualidad establecen fuertes vínculos (Sowell, 2002), y ello hace que la confianza en «Dios» o en la existencia de un ser superior adquiera una sustantiva importancia.

En atención a estas ideas y definiciones, hay que aclarar que los oficios para el cuidado de la vida, que desarrollan los campesinos sanadores han de ser comprendidos en el contexto del curanderismo como una «práctica social curativa tradicional equivalente a un modelo médico alternativo subordinado» (Campos Navarro, 1997). Ante la consolidación de un sistema médico hegemónico, los sanadores han configurado modos subalternos de agencia y resistencia, gracias a los cuales propician condiciones de vida más dignas para los seres con los que comparten sus existencias. Si bien este modelo médico alternativo subordinado tiene sus fundamentos en la observación y la experiencia, también se enriquece con elementos allegados de diferentes tradiciones, los cuales le otorgan un carácter dinámico y una continuidad en el tiempo, debido a los procesos de producción, circulación y apropiación de saberes 5 .

En palabras de Douglas Sharon:

El curanderismo representa un sistema abstracto de conocimiento mágicoreligioso firmemente arraigado [...] que manifiesta una notable capacidad de adaptación. [...] el curanderismo representa no tanto una reacción nativa al catolicismo cuanto un verdadero sincretismo — esto es, una síntesis funcional de las formas religiosas aborígenes y católicas con una gran tenacidad y estabilidad. En suma, el curanderismo parece representar una mezcla de

5 Roberto Campos-Navarro propone dos formas de curanderismo que tienen que ver con los contextos en los cuales este se agencia. Por un lado, encontramos el curanderismo doméstico (o medicina doméstica), que se corresponde con la atención brindada por algún integrante del grupo familiar - abuelo, abuela, padre, madre, hermanos a otro integrante del mismo grupoy el curanderismo especializado, al cual pertenecen «chamanes, parteras, hueseros y sobadores, yerbateros, espiritualistas, fidencistas, médiums y espiritistas» (Campos-Navarro, 1997, p. 11). 
arquetipos comunes a la experiencia religiosa cristiana e indígena [y negra]; en verdad, una forma religiosa mestiza (1980, p. 15).

Y este es otro de los rasgos más importantes de los sanadores. Ellos son sujetos mestizos, herederos de comunidades indígenas, comunidades negras y comunidades europeo-españolas. Sin embargo, a pesar de la fuerza y la imposición del patrón aculturador de origen europeo-español, estos hombres y mujeres le han dado continuidad a su vínculo con la tierra - materializada en las plantas, los animales y los minerales - y la magia, y han encontrado en estas los fundamentos y los elementos para llevar a cabo las prácticas a través de las cuales cuidan de la vida en todas sus formas. De tal modo, a pesar de su condición subordinada, estos campesinos son capaces de agenciar un diálogo de saberes y propiciar la puesta en marcha de formas de medicinas alternativas y complementarias.

Ya que se presentó al curanderismo como el fenómeno cultural en el cual se inserta el oficio de los sanadores, en atención a las comprensiones derivadas del trabajo investigativo, es importante incluir el otro concepto que motiva la escritura de este texto, es decir, el de artesanos de la salud. Esta noción se desprende de los códigos etic, esto es, de las interpretaciones que emanaron del proceso de sistematización de los datos recogidos en el trabajo de campo. Vale la pena aclarar que con ella no se pretende invalidar las ideas presentadas anteriormente acerca del concepto sanador, sino que se reconoce como una posibilidad derivada del ejercicio analítico de la investigación. En correspondencia con el título, se asume a los campesinos participantes como sanadores, tal y como ellos se autodenominan y, a la vez, como artesanos de la salud y la vida.

Según narra la mitología griega, la primera imagen de artesano llegó del dios Hefesto gracias a las enseñanzas obtenidas en la isla de Lemnos, lugar en el que fue criado por las Nereidas luego de ser expulsado del Olimpo. Este dios aprendió las técnicas de la artesanía, que le dieron un lugar privilegiado entre los dioses y los humanos, pues, por su pericia, no tenía parangón alguno. Hefesto, además de artesano, también era herrero, por eso, gracias a la conexión entre sus manos y su pensamiento, logró forjar los más impactantes artilugios para la guerra, así como los más destacados objetos para la vida cotidiana. Todo esto en conjunto le otorgó un lugar destacado en el Olimpo, así como le propició un reconocimiento permanente en la tierra, y ello hizo que fuera invocado para inspirar a todos aquellos seres humanos que asumían el desafío de crear y transformar con sus propias manos el mundo en el que se encontraban. 
Esta deidad del Monte Sagrado es la que inspira para destacar la condición de hacedores de aquellos sanadores que fueron presentados en la primera parte del texto. Y no inspira porque en los testimonios haya sido mencionado y muchos menos porque en sus oficios sea invocado para recibir instrucción; se acude a su imagen porque, como deidad, expresa una de las condiciones más importantes para la realización de las prácticas a través de las cuales se cuida la vida. Para los campesinos, aquello que saben hacer para propiciar la salud y el bienestar es, en realidad, un conocimiento y una inspiración entregados por la divinidad; dicho de otra manera, es un don que su dios les entregó para servir a los otros, de aquí que muchos de ellos también se reconozcan como sanadores y como servidores.

Se propone el concepto de artesanos de la salud para hacer referencia a aquellos hombres y mujeres que, en las montañas, los valles y las selvas, articulan sus manos, sus cabezas y sus corazones con el propósito de brindar un servicio a los otros, a través del cual encuentren vías de recuperación y mejoramiento de sus situaciones de padecimiento. Lo anterior quiere decir que estos sujetos realizan un trabajo que es manual, pero, al mismo tiempo, un trabajo que tiene origen en la necesidad y el deseo de ayudar, y que responde a unas intencionalidades y fines prácticos concretos enmarcados en lo que se podría llamar efectividad. Esta idea es inspirada en los aportes de Sennet (2009), quien señala que, en los oficios de los artesanos, las manos y el pensamiento se integran de forma indisoluble o, como bien lo expresaría Pellicer, estos sujetos encarnan al hombre colectivo «que piensa con las manos» (1995, p. 17).

De tal suerte,

[...] el artesano explora estas dimensiones de habilidad, compromiso y juicio de una manera particular. Se centra en la estrecha conexión entre la mano y la cabeza. Todo buen artesano mantiene un diálogo entre unas prácticas concretas y el pensamiento; este diálogo evoluciona hasta convertirse en hábitos, los que establecen a su vez un ritmo entre la solución y el descubrimiento de problemas (Sennet, 2009, p. 12).

Tal y como se asume en este texto, los artesanos de la salud, además de articular las manos y el pensamiento en su hacer, también implican el corazón. Es en la tríada de manos, pensamiento y corazón donde se comienza a gestar el oficio terapéutico de estos hombres y mujeres. Las manos aluden al contacto, la exploración, la examinación, el diagnóstico; el pensamiento o la cabeza se relacionan con las formas de razonamiento y explicación a través de las que se toman decisiones y se escogen vías de intervención, y el corazón representa la 
opción por el servicio y, en la perspectiva de Boff (2014), los vínculos afectivos de cuidado, que se establecen entre estos agentes de la salud y los usuarios que los buscan. Lo anterior obliga a tomar distancia de cualquier consideración de los artesanos como operarios de una técnica y, al contrario, esta sinergia de manos, pensamiento y corazón hace verlos como hacedores reflexivos, cuyos oficios no se centran en la repetición sino en la creación y la transformación, pues ellos «representan la condición específicamente humana del compromiso» (Sennett, 2009, p. 17), es decir, del servicio a los otros.

Estas últimas ideas retoman una consideración del artesano en general y de los artesanos de la salud en particular, por ello, al inicio de esta parte del texto se consignó uno de los testimonios de los participantes en la investigación, el cual asumía que los campesinos eran las personas más sabias, en especial porque su sabiduría emanaba de su conexión con todo lo que los rodea y con todo lo que experimentan, disfrutan y hasta sufren. Así, se hace referencia a los artesanos del medio ambiente; a los alfareros que le dan continuidad a la vida a través de la labor incansable de las manos, gracias al cuidado de los animales y las plantas; a los trabajadores manuales que encuentran en sus oficios vías concretas para esculpir su propia vida y ayudar a otros a hacer lo mismo, porque saben que estas son obras de arte. En fin, en este texto se propone una comprensión de los artesanos como artífices de nuevas relaciones entre las personas y el mundo, y no solo como operarios que se dedican a crear objetos e instrumentos o a esculpir determinados cuerpos de materia amorfa.

Las manos son fundamentales para agenciar las formas de cuidado, pues son los vehículos a través de los cuales el artesano se vincula con los usuarios de los procedimientos curanderiles; del mismo modo, con estas se da el uso de instrumentos y recursos terapéuticos necesarios en las diferentes intervenciones.

Con las manos se seleccionan, recogen y preparan las plantas para la realización de bebidas, emplastos y unturas; con las manos se construyen los escenarios rituales en los cuales suceden las prácticas: se disponen las luces, el agua, las flores, las botellas de colores, las imágenes de la religiosidad popular, entre otros asuntos; con las manos se hace el diagnóstico de las partes del cuerpo, que se hallan dislocadas y afectadas, y se da lugar a su respectiva reubicación; con las manos se le ayuda a las mujeres a dar a luz, se recibe al infante recién nacido y se le corta el cordón umbilical que lo une a la madre; con las manos se comparte energía vital ya sea a través de su imposición o del tacto curativo; con las manos se hacen sonar las campanas para convocar a las Ánimas del Purgatorio y para instar a los seres humanos a ofrecer una oración por ellas. Como se puede ver, 
las manos están en el centro de las terapéuticas, pero siempre en sinergia con una intencionalidad — que obedece al campo del pensamiento-, y determinadas por un vínculo con el otro y lo otro — que está regido por el ámbito del corazón-.

Ahora bien, como una derivación de las anteriores ideas acerca de los artesanos de la salud, en lo que sigue se exponen unos descriptores de los oficios de estos sujetos. Cabe aclarar que las siguientes líneas son elementos emergentes que se proponen en sintonía con algunas perspectivas aportadas por Sennet (2009) en lo atinente al artesano:

* El artesano de la salud como sujeto de la experiencia: dentro del hacer de los artesanos de la salud, la experiencia se presenta como la vía a través de la cual se enriquecen los saberes y se cualifican los oficios. En palabras de Gadamer, la experiencia es la vía que conduce hacia la sabiduría, así pues «[...] cuanto más domine alguien su poder-hacer tanto mayor será su libertad respecto de ese poder-hacer» (1996, p. 35). Con la experiencia se reconocen los relieves de las prácticas y, gracias a esta, se pueden advertir algunas de las peripecias que trae consigo el ejercicio de un saber en un contexto determinado.

Al hablar de la experiencia, se reconoce que, en la base de toda interacción con las terapéuticas de la salud, el artesano siempre está en un permanente ejercicio de sus saberes, como una condición sine qua non para adquirir la maestría necesaria que le otorgue legitimidad y reconocimiento en su propia comunidad. Solo en la medida en que este sujeto ejerce lo que sabe es capaz de ampliar su capacidad de acción y decisión, asunto necesario para responder ante las distintas particularidades de su labor. De la misma manera, gracias al ejercicio continuado del oficio se amplía la red de conocimientos, explicaciones y comprensiones alrededor de los desafíos procedimentales de una tarea que implica la atención de la salud.

Tal y como muestra Sennet, «[...] toda artesanía se funda en una habilidad desarrollada en alto grado. De acuerdo con una medida de uso común, para producir un maestro carpintero o músico hacen falta diez mil horas de experiencia» (2009, p. 18). De la misma manera, además de las vías de iniciación que se reconocieron en la investigación, tales como (i) enfermedades, dolencias o traumatismos propios del sanador o de personas de su familia o cercanas a este; (ii) instrucciones recibidas de otro sanador de su comunidad o de algún familiar; (iii) escucha de relatos de personas mayores a través de la transmisión oral; (iv) sueños iniciáticos; (v) aproximación a materiales diversos como libros o contenidos de otras formas religiosas y espirituales, y (vi) instrucción a través del contacto con un profesional de la salud o con personas que tienen proximidad a este campo; los 
artesanos de la salud necesitan poner en escena sus oficios, de modo constante, para materializar su real condición de agentes comunitarios de salud.

De esto da cuenta José Leonardo cuando afirma que, a lo largo de su tarea como componedor, ha «arreglado más de cincuenta rodillas». La primera situación, en la que intentó reubicar el menisco de su compañera, falló, pero después de la instrucción recibida del ortopedista, asumió el desafío de repetir el procedimiento en el cual no había sido efectivo y, gracias al éxito de esta segunda intervención, adquirió la confianza para reubicar cantidad de meniscos, así como para incursionar en la desubicación de otros huesos y tendones de las personas que comenzaron a buscarlo.

El caso de Blasina del Socorro es similar. Su ejercicio de componedora o sobandera comenzó con la ayuda brindada a un caballo y a una ternera. Posteriormente, dado que el resultado fue satisfactorio, ella hizo extensiva su atención a personas de San José de la Montaña, municipio en el que es una de los pocos sobanderos que se mueve entre las veredas y el casco urbano, para atender a quienes requieren su ayuda.

En este primer descriptor se retoman estos dos casos, pues sirven de ejemplos para ampliar aquello a lo que se refirió Sennet sobre las «diez mil horas de experiencia». No se sabe, al final, cuántas han sido las horas para alcanzar el dominio de la práctica, sin embargo, sí se pudo ver que, a través de las sinuosidades del oficio, se fortaleció un saber-hacer y la misma capacidad de decisión.

Estos dos casos hablan de un recorrido, de unas trayectorias, de unos caminos... Estos siempre serán distintos y variados; no siempre serán lo mismo y expresarán formas diversas de operar el saber. Y estos caminos remiten a un asunto que ya antes se había mencionado sobre los artesanos de la salud como hacedores reflexivos, que fundamentan sus oficios en el compromiso de servir a otros gracias al ejercicio de sus capacidades y saberes. Entonces, «el artesano [de la salud] es más que un técnico» (Sennet, 2009, p. 18), esto es, más que un operario, un reproductor o un repetidor de procedimientos.

Cada caso de artesanía de la salud supone una actualización de conocimientos y una ampliación de las redes de significado que hacen parte de la experiencia; a la par, gracias a las prácticas se pueden transformar representaciones e incorporar cavilaciones acerca de las formas del hacer, con el fin de enriquecerlas y actualizarlas. La producción no se da de forma seriada, es más, a pesar de hablar del artesano, las prácticas para el cuidado de la vida no dependen, en estricto sentido, de la labor del agente de la salud que las pone en escena; los usuarios también se vinculan al ejercicio y aportan sus propias interpretaciones y consideraciones. 
* El artesano de la salud ofrece su servicio a la comunidad y esta valida su saber-hacer: otro de los rasgos de los artesanos de la salud tiene que ver con la importancia de la comunidad para la refrendación de sus oficios. No es fácil encontrar a un artesano de la salud por fuera de un grupo humano que otorga importancia y trascendencia a su oficio. Igualmente, muchas comunidades campesinas se articulan alrededor de los desempeños de determinados sujetos, que son reconocidos por sus aportes a la vida en colectivo, por ello, es muy complejo dimensionar la vida comunitaria sin la agencia de los seres humanos que aportan sus trayectorias y sistemas de conocimiento alrededor del territorio, lo religioso, las relaciones sociales y, para el caso, las formas de atención de la salud.

A propósito del dios Hefesto, Sennet (2009) recuerda que «[...] un antiguo ideal de la artesanía [...] unía habilidad y comunidad» (p. 38). Era y es la comunidad el escenario expedito en el cual suceden las interacciones entre los agentes y los usuarios de la salud; es más, este espacio colectivo se construye gracias al desarrollo de relaciones sociales y en este, alrededor del cuidado de la vida como un saber-hacer, se establecen vínculos de confianza y de credibilidad frente a lo que se ofrece como servicio terapéutico. Por ello, se puede afirmar que los artesanos de la salud son sujetos que participan de comunidades de aprendizaje más amplias, en las que el ethos de hacedores reflexivos les brinda autoridad y legitimidad.

En los casos de los ocho participantes de la investigación, ellos gozan de un reconocimiento en sus comunidades, asunto que destaca la importancia de sus prácticas para atender distintas afectaciones en la salud. Se pudo llegar hasta los hogares de estas personas gracias a las referencias dadas por otros coterráneos sobre ellas mismas, sobre sus modos de llevar a cabo diagnósticos y análisis de los estados de salud y sobre las terapéuticas concretas para brindar atención. Por esta razón, el primer contacto con las prácticas estuvo precedido por una escucha de diferentes relatos de experiencia y descripciones de procedimientos.

Este reconocimiento se cimienta sobre una premisa fundamental: el artesano de la salud reconoce que su oficio es perfectible, por ello, siempre está buscando las alternativas para mejorarlo. En otras palabras: la artesanía es, en sí misma, una habilidad para hacer bien hechas las cosas. Al respecto, Sennet señala que:

Es posible que el término «artesanía» sugiera un modo de vida que languideció con el advenimiento de la sociedad industrial, pero eso es engañoso, «artesanía» designa un impulso humano duradero y básico, el deseo de realizar bien una tarea, sin más. La artesanía abarca una franja mucho más amplia que la correspondiente al trabajo manual especializado. Efectivamente, es 
aplicable al programador informático, al médico y al artista; el ejercicio de la paternidad, entendida como cuidado y atención de los hijos, mejora cuando se practica como oficio cualificado, lo mismo que la ciudadanía (Sennet, 2009, p. 12).

Como se ha intentado destacar, el reconocimiento del artesano de la salud tanto como oficio y como categoría, que supone un saber-hacer y un hacer-bien, más que el resultado de un asunto azaroso, es una expresión de la circulación, la apropiación y el uso de saberes alrededor de modos concretos de entender y explicar las etiologías de las enfermedades y las epidemiologías socioculturales. Al respecto, la legitimidad dada por los coterráneos frente al hacer de los campesinos sanadores tiene que ver con que estos últimos son sujetos que también forman parte de las comunidades, comparten sus problemáticas, conocen sus necesidades y ponen ello en función de una serie de procedimientos que gozan de una eficacia simbólica e instrumental. Y este último asunto tiene raigambre en procesos de observación, experimentación, conjetura y análisis del desarrollo de los procedimientos curanderiles. Esto demuestra que no se trata de la repetición de oficios, sino de la reinvención de modos de comprensión del ser humano en sus diferentes dimensiones.

* El hogar-taller es el espacio de creación del artesano de la salud: el artesano requiere un lugar concreto para llevar a cabo su actividad creadora. Las tareas que este sujeto realiza no suceden, exclusivamente, en el mundo de las ideas ni en el plano inmaterial, al contrario, dada la condición manual de su oficio, estas demandan la existencia de un espacio en el que aquella sinergia entre lo que piensa, lo que desea y lo que hace que tenga lugar.

El taller es, por excelencia, el lugar en el que este hacedor reflexivo despliega todo su potencial para intervenir la realidad y para reinventarla conforme a sus conocimientos y al desarrollo de procedimientos específicos. Sin el taller no podría pensarse la existencia del artesano y, sin el artesano, el taller no tendría importancia, pues sería un espacio vacío. Por esta razón, para comprender las sinuosidades del oficio de los artesanos de la salud, hay que develar las particularidades del lugar en el que llevan a cabo a sus prácticas y en el que establecen vínculos de servicio con los usuarios.

Frente a este asunto:

El taller es el hogar del artesano, expresión que debe entenderse históricamente en su sentido literal. En la Edad Media, los artesanos dormían, comían y criaban a sus hijos en los lugares en los que trabajaban. El taller, como tal y como hogar de las familias, era de escala reducida, pues cada uno alber- 
gaba como máximo unas pocas docenas de personas [...] Es fácil comprender el atractivo romántico que el taller-hogar ejercía [...] un espacio de trabajo humano, donde también parecían encontrar un buen hogar, un lugar en el cual el trabajo y la vida se entremezclaban (Sennet, 2009, p. 39).

En lo atinente a los artesanos de la salud del Norte de Antioquia, se repite la situación descrita por este teórico del hacer manual. En la investigación, los hogares de todos los participantes se transformaron en los centros de operación; así, las cocinas, las salas, las habitaciones o los corredores de las casas son los lugares privilegiados para intercambiar diferentes palabras acerca de los padecimientos experimentados, para proceder con los procesos diagnósticos y para, finalmente, llevar a cabo la intervención alrededor de la consolidación de espacios rituales.

No obstante, los hogares de los usuarios también pueden transformarse en los talleres de los artesanos, pues, cuando uno de los consultantes no tiene la posibilidad de desplazarse por sus condiciones físicas (personas postradas que no podían caminar, personas de edad avanzada, personas con afectaciones en la estructura osteomuscular), hasta su hogar llega el sanador con sus instrumentos y materiales. Este espacio de los otros acoge al artesano y le permite cumplir con las tareas para las cuales fue procurado. Además, la consulta deriva en una conversación mediada por el compartir de un tinto, una aromática, alguna comida o por el intercambio de algún producto derivado del trabajo agropecuario. Lo anterior permite comprender que las formas de atención de la medicina tradicional se asemejan más a intercambios familiares fundamentados en la confianza y en la emergencia de la palabra como vehículo de contacto; del mismo modo, esto se articula con la idea que Sennet mencionó en la última línea de la cita: en el taller, trabajo y vida se entremezclan.

Se encuentran así dos formas de hogar-taller: por un lado, el de los agentes de la salud y, por el otro, el de los usuarios de las prácticas. Por lo general, el más frecuentado es el primero, pues, a cualquier hora del día se ve transformado por la presencia de quienes reclaman alguna asistencia; empero, así como muestra Ezequiel Ander-Egg, en uno u otro hogar-taller «se trabaja, se labora y se transforma» (1991, p. 10), se tejen vínculos y se configura comunidad.

También, se puede ver que dos de los participantes privilegian el hogar de sus consultantes como taller. En concreto, esto hace referencia a los casos de Berta Inés Avendaño Agudelo y de José David Múnera Ochoa. Como Berta Inés era la partera que invitaban para cuidar y acompañar los partos, ella debía trasladarse hacia las casas de las mujeres parturientas para estar allí durante el alumbramiento y el puerperio. En este caso, las camas se transformaban en las 
camillas hospitalarias, las habitaciones en los centros de operación y la sala o la cocina en los espacios de espera de los familiares. En lo atinente a José David, dada la resistencia que generaban, al inicio, sus oficios entre sus familiares, él decidió que los lugares de atención de los usuarios serían sus propias casas y, por ello, luego de concertar una cita, él llega hasta allí, realiza la práctica curanderil y aprovecha para hacer una limpieza energética del espacio físico.

Otro aspecto importante, que ha de vincularse a la existencia del hogar-taller o del taller, tiene que ver con el uso de materiales e instrumentos. Según señala Sennet, «[...] el pasado de la artesanía y de los artesanos también sugiere maneras de utilizar herramientas, organizar movimientos corporales y reflexionar acerca de los materiales, que siguen siendo propuestas alternativas viables acerca de cómo conducir la vida con habilidad» (2009, p. 14). Dado que estas formas de medicina tradicional tienen toda una configuración manual y simbólica, la manipulación de diferentes instrumentos se transforma en un imperativo, pues sin los recursos técnicos y de operación sería imposible el desarrollo de los oficios.

Así las cosas, en el caso de Martha Genoveva, al desarrollar oficios relacionados con la herbolaria y la heliocromoterapia, recurre al uso de plantas, botellas de vidrio, agua y a la fuerza del sol; Jesús María, como animero, recurre a la campana como instrumento sagrado para invitar a las Ánimas del Purgatorio a pasear por las calles del pueblo y para vincular a los seres vivos en la oración por estos seres; Blasina del Socorro y José Leonardo, al ser los sobanderos o los componedores, recurren al uso de sus manos para palpar, diagnosticar y reubicar, empero, la mayoría de veces esta intervención implica el uso de aceites, cremas o líquidos que ayuden a deslizar las manos y, además, se acompaña de la recomendación de plantas medicinales para desinflamar, cicatrizar y agilizar los procesos de recuperación.

Por su parte, Berta Inés, gracias a la capacitación recibida por los profesionales de la medicina alopática o facultativa, recibió un baúl con diferentes materiales para atender los partos: tijeras, pinzas, mechero, delantal, jabón, cuerdas, alcohol, entre otros. Mas, dada su formación inicial como partera tradicional, ella también usa plantas medicinales para generar tranquilidad y para apoyar los procesos de desinflamación o cicatrización de heridas derivadas del parto. En lo atinente a José David, para la realización de la limpieza a través de la luz, él solicita una fotografía a sus consultantes o, si estos no la tienen, usa una hoja de block para hacer el registro del aura y, gracias a la luz de una vela, adelanta los procesos diagnósticos. En el caso de Argemira, el agua, la luz, el alcohol y el aceite son fundamentales para realizar la oración y canalización de José Gregorio 
Hernández; sin estos materiales no hay procedimiento curanderil. Y, finalmente, Ramiro Antonio vuelve al uso de las manos para transmitir fuerza y vitalidad a las personas convalecientes que lo buscan.

En sintonía con todo lo dicho, el oficio de los artesanos de la salud, en sus respectivos talleres, demanda el uso cuidadoso y reflexivo de recursos y materiales terapéuticos de muy diverso orden.

* El artesano de la salud desarrolla su oficio pensando en el servicio y no en la transmisión o la transferencia: uno de los asuntos más interesantes derivados de los ejercicios de conversación con los campesinos participantes tuvo que ver con que ellos no les daban importancia a los procesos de transmisión de sus saberes y oficios. Dado que la mayor parte de sus vías de iniciación como artesanos de la salud no estuvieron articuladas a eventos de enseñanza y aprendizaje con un mentor, un maestro o un familiar, en el momento de indagar por la continuidad de sus terapéuticas expresaron que este asunto dependía de la vocación de servicio de las personas y no del establecimiento de un vínculo específico para aprender. En otras palabras, su finalidad no era la consecución y la preparación de diferentes discípulos o aprendices, pues ese no fue su caso para incursionar en el mundo del curanderismo mestizo.

Quizá este asunto difiera de lo expresado por Sennet, para quien la formación técnica del artesano implica «el contacto directo con los instrumentos y la explicación oral transmitida de generación en generación» (2009, p. 53). La transferencia de habilidades, entonces, no está en el centro de las preocupaciones, pero lo que sí se reconoce es el desarrollo de procesos reflexivos acerca de lo que se hace y la necesidad y la obligatoriedad de hacer las cosas bien. Al respecto, el siguiente testimonio amplía estas ideas:

Si Dios se los da es que eso es innato. Lo que yo hago no me interesa enseñar, esa es la equivocación, que supongamos que yo coja a un muchacho de quince años a orientarlo así tenga dones, no se puede, hay que darle pautas, pero no se le puede enseñar, las pautas que hay que enseñarle al que tiene dones y está empezando son: cuidado, analice, fijese... Dios sabe cuándo va dando sus cosas, él es el que sabe y según su corazón. Los dones de Dios son un libro y yo lo abro por la bondad de él (José David Múnera Ochoa).

En relación con este asunto, no se quiere sugerir la idea de que la transmisión y la instrucción de determinados oficios no sean importantes; evidentemente, para la continuidad de ciertos procedimientos esta puede ser una vía concreta, pero, en el caso de los participantes de la investigación, ellos abogan por otras formas 
de contacto y relación con la emergencia de los dones, pues, como se acaba de notar, la interpretación acerca de las formas de curación se explica como una dádiva allegada de la fuerza espiritual. Evidentemente, luego de que diferentes personas despierten sus dones, podrán establecer redes y vías de intercambio con otros sujetos, mientras tanto, a la luz de los testimonios de los artesanos, lo más importante es el reconocimiento de la importancia del gusto y el llamado al servicio, esto es, la emergencia de la vocación.

\section{A MODO DE CONCLUSIÓN}

A propósito de los procesos de atención de la salud y la enfermedad y de la comprensión de los sistemas médicos como sistemas culturales (Langdon, 2014), es importante revisar la manera como la academia $-\mathrm{y}$, en concreto, los investigadores de la salud - se han aproximado y han interactuado con diferentes grupos humanos y sujetos que se desempeñan como agentes comunitarios de salud. La investigación cualitativa ha de generar otras comprensiones alrededor de los conceptos y las categorías que, comúnmente, se utilizan para nombrar la exterioridad y la alteridad.

Y, sin lugar a dudas, esta es la pretensión que ha orientado la proposición de un concepto como el de artesanos de la salud, para hacer referencia a los oficios de hombres y mujeres campesinos, quienes con sus manos se han dedicado a cuidar la vida y atender los padecimientos de otros seres. No se trata, por esta vía, de invisibilizar las propias formas a través de las cuales los sujetos se nombran, sino de ahondar en otras posibilidades y comprensiones de aquello que las personas hacen, en lo que respecta a la salud, en los territorios que habitan.

De tal suerte, al plantear este concepto se está reconociendo que la atención a los procesos de salud y enfermedad, a través de diferentes prácticas para el cuidado de la vida, es un ejercicio artesanal en el que se ponen en escena saberes, experiencias e instrumentos. Se destaca entonces la condición artesanal de prácticas como la herbolaria, el sobanderismo, la partería, la imposición de manos, la limpieza a través de la luz, entre otras, las cuales se erigen sobre la base del cuidado, el reconocimiento y el respeto de las diferentes formas de vida, que se encuentran en esta «casa común» que es la tierra. Desde esta consideración, el cuidado no solo se encarna en la explicación de la existencia y los vínculos de las personas entre sí: este supone un entretejido con tantísimas formas de vida contenidas y expresadas a través del agua, el aire, el fuego y la tierra; de formas de vida expresadas en la diferencia. 
Por esta razón, hablar de artesanos de la salud, que llevan a cabo prácticas para cuidar la vida, invita a descolocar al antropocentrismo como causa, efecto y explicación de la existencia, para, en su lugar, ubicar las relaciones de reciprocidad que se dan entre múltiples formas de vida, dentro de este gran ser viviente que es la Madre Tierra. Y este ha de ser uno de los grandes desafíos de la antropología de la salud: comprender que la sanación —o, si se quiere, el restablecimiento de las dolencias y los padecimientos - ha de implicar unas nuevas relaciones no solo entre los seres humanos, sino entre estos, los territorios que habitan y los diferentes seres vegetales, animales y minerales que también forman parte de ellos.

\section{REFERENCIAS}

Ander-Egg, Ezequiel (1991). El taller: una alternativa de renovación pedagógica. Buenos Aires: Magisterio del Río de la Plata.

Armus, Diego (2002). Cultura, historia y enfermedades. A modo de introducción. En D. Armus (comp.), Entre médicos y curanderos. Cultura, historia y enfermedad en la América Latina moderna (pp. 11-25). Buenos Aires: Norma.

Bajtin, Mijail (1998). Estética de la creación verbal. México: Siglo XXI.

Boff, Leonardo (2014). Saber cuidar: ética do humano - compaixão pela terra. Petrópolis: Vozes.

Campos Navarro, Roberto (1997). Nosotros los curanderos. México: Nueva Imagen.

Campos Navarro, Roberto (2016). Doña Rosita Ascencio, curandera purépecha. México: Artes de México.

Ceballos Gómez, Diana Luz (1994). Hechicería, brujería e inquisición en el Nuevo Reino de Granada. Un duelo de imaginarios. Bogotá: Universidad Nacional de Colombia.

Ceballos Gómez, Diana Luz (2002). «Quyen tal hace que tal pague» Sociedad y prácticas mágicas en el Nuevo Reino de Granada [1999]. Bogotá: Ministerio de Cultura.

Gadamer, Hans-Georg (1996). El estado oculto de la salud. Barcelona: Gedisa.

González Echevarría, Aurora (2009). La dicotomía emicletic. Historia de una confusión. Barcelona: Biblioteca A/Sociedad, Anthropos.

González Rodríguez, Adolfo León (2005). Educación y práctica médicas en Antioquia. Antecedentes históricos de la fundación de la Escuela de Medicina de la Universidad de Antioquia. Iatreia, 18(3), 332-343. 
Harris, Marvin (1982[1979]). El materialismo cultural. Madrid: Alianza Editorial.

Langdon, Esther Jean (2014). La negociación de lo oculto: chamanismo, medicina y familia entre los siona del bajo Putumayo. Popayán: Universidad del Cauca.

López, Lucero, Nhora Cataño, Heddy López y Vilma Velásquez (2011). Diversidad cultural de sanadores tradicionales afrocolombianos: preservación y conciliación de saberes. Aquichán, 11(3), 287-304.

Mallimaci, Fortunato y Verónica Giménez Béliveau (2006). Historia de vida y métodos biográficos. En Irene Vasilachis de Gialdano (coord.), Estrategias de investigación cualitativa (pp. 175-212). Barcelona: Gedisa.

Mockus Sivickas, Antanas (1994). Anfibios culturales, moral y productividad. Revista Colombiana de Psicología, 3, 125-135.

Módena, María Eugenia (1990). Madres, médicos y curanderos. Diferencia cultural e identidad ideológica. México: Ciesas.

Múnera, Mauricio (2017). Saberes y prácticas campesinas de sanación: una aproximación a la medicina tradicional en el Norte de Antioquia, Colombia. Pensamiento Actual, 17(29), 11-25.

Múnera, Mauricio (2019). Las metodologías biográficas y narrativas y sus aportes para el desarrollo de procesos antropológicos alrededor de la salud y la enfermedad en comunidades campesinas. Faeeba, 28(56), 70-89.

Pellicer, Jorge (1995). Artesanos del porvenir. México: Secretaría de Educación Pública/ Universidad Autónoma Metropolitana.

Press, Irwin (1971). The urban curandero. American Anthropologist, 73, 741-756.

Sennett, Richard (2009). El artesano. Barcelona: Anagrama.

Sharon, Douglas (1980). El chamán de los cuatro vientos. México: Siglo XXI.

Sowell, David (2002). Andanzas de un curandero en Colombia: Miguel Perdomo Neira y «la lucha entre el buen sentido y la ignorancia ciega». En Diego Armus (comp.), Entre médicos y curanderos. Cultura, historia y enfermedad en la América Latina moderna (). Buenos Aires: Norma.

Spector, Rachel E. (2003). Las culturas de la salud. Barcelona: Prentice Hall.

Zolla, Carlos (1986). Terapeutas, enfermedades y recursos vegetales. México indigena, 9, 16-20. 